\title{
Enriquecimiento forestal en fajas en un bosque explotado del Chaco húmedo
}

\author{
Enrichment planting in lines on exploited forest of humid Chaco
}

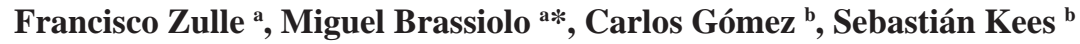 \\ *Autor de correspondencia: ${ }^{a}$ Universidad Nacional de Santiago del Estero, Facultad de Ciencias Forestales, INSIMA, \\ Av. Belgrano (S) 1912, Santiago del Estero, Argentina, tel.: +54-385-5800007, mikyb@unse.edu.ar \\ b INTA, Estación Forestal Presidencia de la Plaza, Argentina.
}

\begin{abstract}
SUMMARY
In the province of Chaco, native forests cover five million hectares approximately, but over-exploitation of these forests has caused an alarming decrease in the participation of commercially important species. This project evaluated a technique for enrichment in a parcel of forest of humid Chaco that was heavily exploited during last century until the '30s and with some mild extractions after that. At the moment of establishing the evaluation, the woodland had $17 \mathrm{~m}^{2}$ of basal area. The survival of planted individuals, the competence situation in which they were developing and their growth in height and diameter were assessed. Six years after planting, findings showed that only in the case of Peltophorum dubium surviving results were good and that the level of growth, both in diameter and in height, was low because the plants of enrichment grew under strong competence. The individuals under specific situations of minor competence marked differences in growth, compared with the rest.
\end{abstract}

Key words: forest enrichment, forests recovery, humid Chaco.

\section{RESUMEN}

En la provincia del Chaco, el bosque nativo cubre aproximadamente cinco millones de hectáreas, pero la sobreexplotación del mismo ha ocasionado que la participación de las especies comercialmente importantes disminuya en forma alarmante. Este trabajo evaluó una técnica de enriquecimiento en fajas con plantas de Grevillea robusta, Handroanthus heptaphyllus y Peltophorum dubium en un bosque del Chaco húmedo que fue explotado en el siglo pasado hasta la década del treinta y con algunas extracciones leves posteriores. Al momento de establecer el ensayo, la masa contaba con $17 \mathrm{~m}^{2}$ de área basal (57 \% del área basal del bosque sin aprovechamiento). Se evaluó la sobrevivencia de los individuos plantados, la situación de competencia en la cual se desarrollaban y su crecimiento en diámetro y altura. Los resultados, luego de seis años de la plantación, mostraron que sólo en el caso de Peltophorum dubium se obtuvieron buenos resultados de sobrevivencia y que el crecimiento, tanto en diámetro como en altura fue pobre, ya que las plantas de enriquecimiento crecieron bajo fuerte competencia. Los individuos que encontraron situaciones puntuales de menor competencia marcaron diferencias en crecimiento frente al resto.

Palabras clave: enriquecimiento forestal, recuperación de bosques, Chaco húmedo.

\section{INTRODUCCIÓN}

En la provincia del Chaco, el bosque nativo cubre casi cinco millones de hectáreas. El uso forestal irracional y la creciente expansión de la frontera agrícola provocan año tras año la degradación y desaparición de miles de hectáreas de bosques nativos. Esta situación de progresiva degradación del bosque como recurso económico por el uso forestal no planificado, se agrava por la superposición con la actividad ganadera extensiva dentro del bosque, que dificulta la regeneración natural y el desarrollo de renovales. Dependiendo del ritmo y de la intensidad de las explotaciones anteriores resulta en superficies prácticamente desarboladas o en bosques degradados sin potencial para un manejo forestal (Grulke et al. 2007). El inventario de la provincia del Chaco muestra que la participación de las especies comercialmente importantes disminuye en forma alarmante. Esto define la necesidad de adoptar medidas de manejo orientadas a aumentar la participación de especies comerciales en el bosque (Gobierno de la Provincia de Chaco 2006).

Una forma de revertir dicho proceso consiste en el aumento de la participación de especies de alto valor económico en la estructura de estos bosques mediante la técnica de enriquecimiento (Lamprecht 1990, Grulke et al. 2007, Brassiolo et al. 2013).

La mayoría de las experiencias de esta técnica silvicultural en Argentina se han concentrado en la selva misionera (Eibl et al. 1993, Sánchez et al. 1993, Montagnini et al. 2006). Para la región chaqueña húmeda, la mayoría de los 
trabajos realizados apuntaron a evaluar el crecimiento de los individuos plantados, por lo que se dispone de datos de crecimiento en enriquecimientos en fajas y en bosquetes con Peltophorum dubium (Spreng.) Taub. (Gómez y Cardozo 2003, Senilliani et al. 2006).

Dado el escaso conocimiento sobre el desempeño de la técnica de enriquecimiento en fajas en ambientes del Chaco húmedo y reconociendo la importancia de comprender este proceso, los objetivos de este trabajo son: 1) evaluar la sobrevivencia y los daños observados en individuos de diferentes especies valiosas plantadas en fajas de enriquecimiento; 2) evaluar la influencia de la competencia y de la orientación de las fajas de enriquecimiento sobre el crecimiento en altura y diámetro de plantas de Peltophorum dubium.

Si los individuos plantados presentan buena sobrevivencia, tal como se espera, las fajas de enriquecimiento con orientación E-O, al presentar mejor insolación, deben influir positivamente en el desarrollo en diámetro y altura de las plantas de enriquecimiento.

\section{MÉTODOS}

Ubicación del estudio. El trabajo se llevó a cabo en la Estación Forestal Plaza, campo anexo de la Estación Experimental Agropecuaria INTA Sáenz Peña, ubicada a los $59^{\circ}$ $48^{\prime}$ de longitud $\mathrm{O}$ y $26^{\circ} 56^{\prime}$ de latitud $\mathrm{S}$, a $75 \mathrm{~m}$ s.n.m., en el extremo oeste del Distrito Chaqueño Oriental (Cabrera 1976) (figura 1). El área estudiada pertenece a la subregión de Esteros, Cañadas y Selvas de Ribera y al tipo forestal Río de Oro, de aproximadamente $16.773 \mathrm{~km}^{2}$ (Morello y Adámoli 1974). Presenta relieve de influencia fluvial, muy joven, elaborado por el sistema de ríos autóctonos de la llanura chaqueña (ríos de Oro, Guaycurú, Cangüí, Tragadero y Negro). El modelo de vegetación es el más heterogéneo del Chaco, con varios tipos de bosques, pastizales y pajonales (Morello y Adámoli 1974).

Clima. El clima es subtropical subhúmedo seco, con precipitaciones superiores en verano-otoño. El promedio anual oscila en $1.100 \mathrm{~mm}$. La temperatura media anual es de $21,4^{\circ} \mathrm{C}$; la temperatura media de invierno es de $15^{\circ} \mathrm{C}$ y la media de verano oscila entre los $27^{\circ} \mathrm{C}$ y $28^{\circ} \mathrm{C}$ (Olivares et al. 1997).

Según el índice de humedad de Thornthwaite, el área se encuentra con menos de $50 \mathrm{~mm}$ de déficit hídrico; aunque pueden ocurrir excesos y deficiencias mayores en diferentes épocas del año. También se presentan años en que la precipitación es excesiva y otros en que el déficit es mayor que el promedio. En términos generales el déficit hídrico es leve de julio a febrero. La frecuencia de heladas meteorológicas es de 8 días/año, siendo el período libre de heladas de 300 a 320 días anuales (Gómez 2010).

Suelo. El suelo pertenece a la serie denominada Martina, clasificados como Natrustalf típico del orden de los Alfisoles, que se encuentra en lomas cerradas evolucionadas, de relieve normal. Tiene un horizonte superficial lixiviado, color gris pardusco claro, textura media; un subsuelo gris oscuro, textura pesada, que descansa sobre un material pardo grisáceo oscuro de textura pesada. Presenta moderado contenido de materia orgánica, buena a alta capacidad de retención de agua hasta los $145 \mathrm{~cm}$ de profundidad estudiados. Son suelos moderadamente profundos con penetración de raíces hasta alrededor del metro (INTA-GPC 1997). Los problemas principales de estos suelos son que carecen de horizonte arable (horizonte A2 en superficie) y son fuertemente sódicos. Es un suelo forestal, que sería aconsejable no desmontar, sino utilizarlo con adecuados turnos de manejo (INTA-GPC 1997).

Vegetación. La vegetación constituye un bosque alto, rico en árboles de madera dura y semidura. El estrato de mayor cobertura mide entre ocho y dieciséis metros. Según Morello y Adámoli (1974), las especies leñosas de mayor importancia son Syagrus romanzoffiana Cham., Ruprechtia polystachya Gris., Phyllostylon rhamnoides (Pois.) Taub., Patagonula americana L., Gleditsia amorphoides (Gris) Taub., Handroanthus heptaphyllus (Vell.) Mattos., Diplokeleba floribunda N.E. Brown, Astronium balansae Engl. y esporádicamente Schinopsis balansae Engl.

El sector del bosque en el que se emplazó el enriquecimiento es un monte alto que fue fuertemente aprovechado en el siglo pasado hasta la década del treinta y soportó algunas extracciones posteriores leves. Al momento de establecer las fajas de enriquecimiento la masa contaba con una densidad poco menor de 300 árboles ha-1 y 16,6 m² ha-1 de área basal, lo que representa el 57 \% del área basal del bosque sin aprovechamiento establecido en el mismo sitio forestal. Las principales especies presentes en el área de estudio fueron Ruprechtia polystachya, Gleditsia amorphoides, Patagonula americana, Caesalpinia paraguariensis (Parodi) Burk., Astronium balansae, Phyllostylon rhamnoides, Diplokeleba floribunda, Acacia praecox Griseb.

Descripción del ensayo. El ensayo fue instalado en la Estación Forestal Plaza, perteneciente al INTA Sáenz Peña en el año 1999 (figura 2). El mismo consta de cuatro parcelas de enriquecimiento en fajas. Originalmente se abrieron fajas de $2 \mathrm{~m}$ de ancho, pero con las limpiezas se fueron ensanchando hasta promediar los 3,5 m, donde se eliminaron los árboles enfermos, mal formados, arbustos y maleza. En cada parcela, las fajas se disponen de forma paralela con un distanciamiento que varía entre 15 y $25 \mathrm{~m}$. El distanciamiento entre plantas es de 2,5 m (200 plantas ha-1), cubriendo una superficie total de 2,4 ha. En cada línea se realizó al menos una limpieza anual de maleza y enredaderas con machete y desmalezadora.

Evaluación del desarrollo de los individuos plantados. La medición del ensayo se realizó durante el invierno del año 2004 (en el mes de agosto) y se consideraron las siguientes variables: 


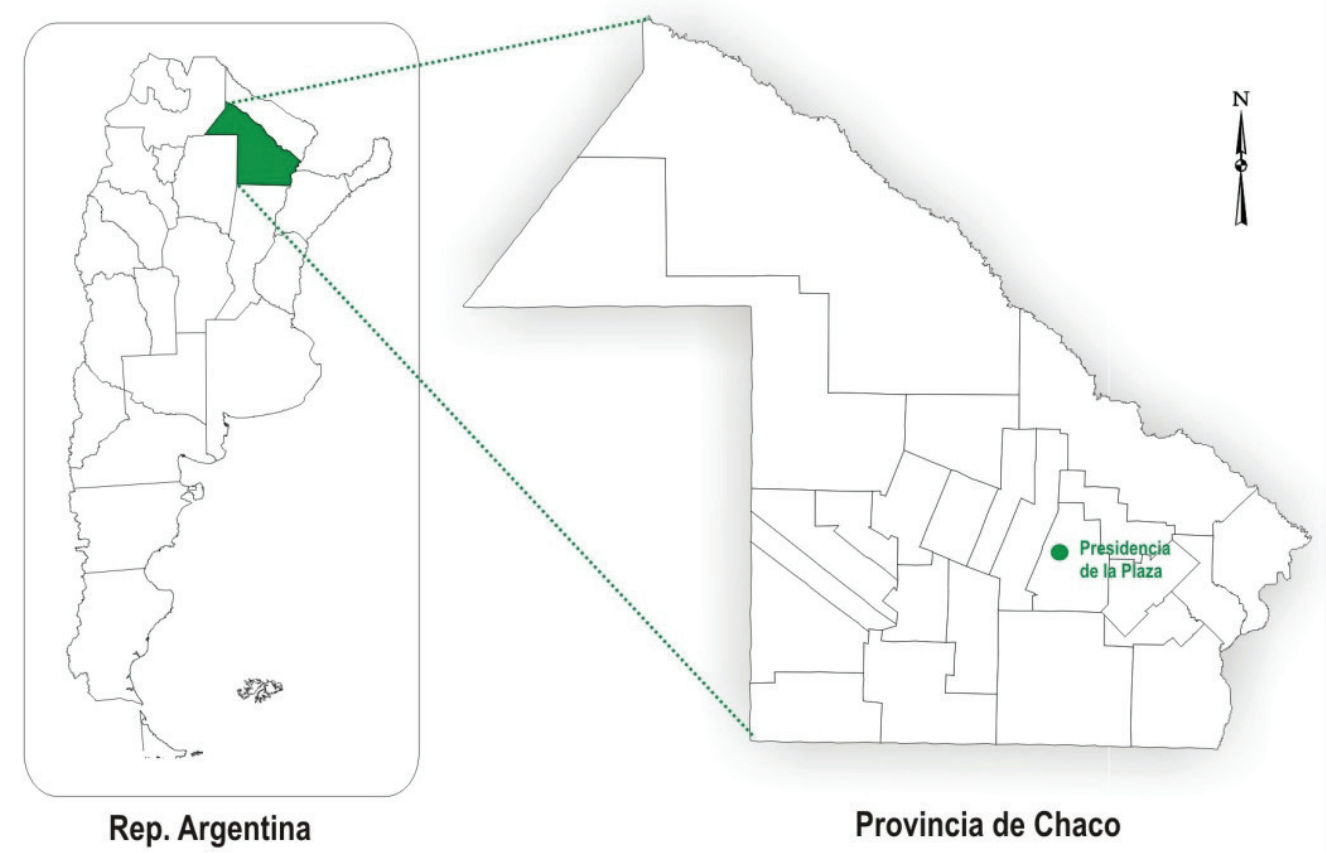

Figura 1. Ubicación del área de investigación.

Location of the research area.

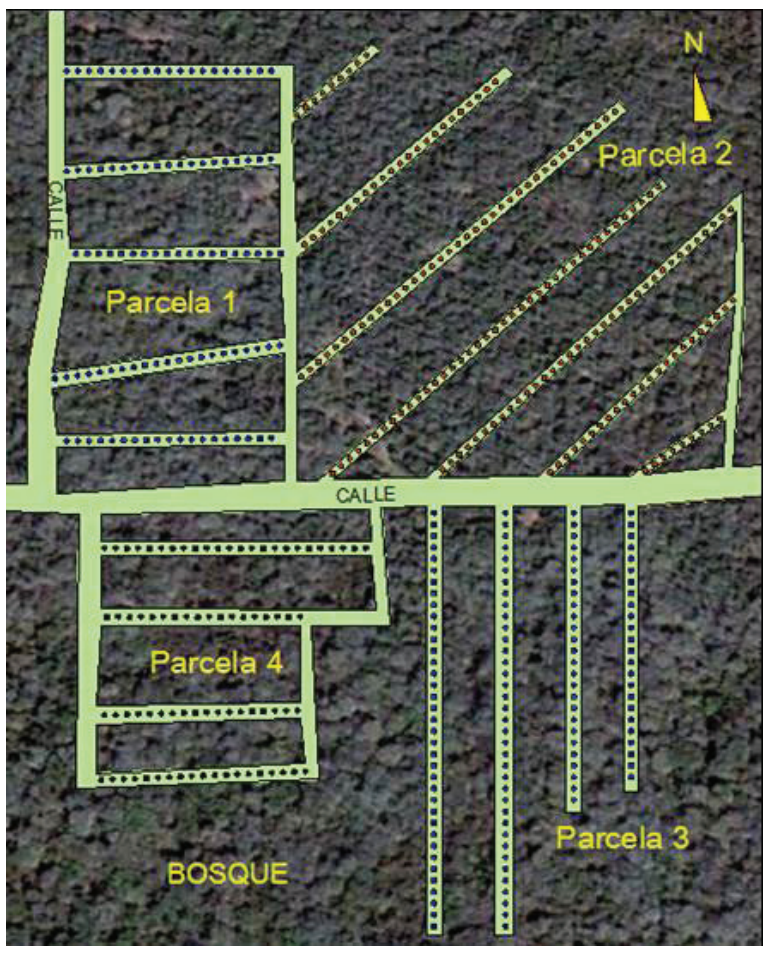

Parcela 1: 94 individuos, en 5 fajas de $50 \mathrm{~m}$ de largo (0,5 ha), plantadas con ibirá pitá guazú (Peltophorum dubium), orientación E-O.

Parcela 2: 183 individuos, en 7 fajas con largos que varían entre $20 \mathrm{~m}$ y $120 \mathrm{~m}$ (0,92 ha), plantadas con grevillea (Grevillea robusta Cunn), orientación NE-SO.

Parcela 3: 103 individuos, en 2 fajas de $93 \mathrm{~m}$ de largo y 2 fajas de $63 \mathrm{~m}$ (0,52 ha), plantadas con ibirá pitá guazú (Peltophorum dubium), orientación N-S.

Parcela 4: 94 individuos, en 2 fajas de $60 \mathrm{~m}$ y 2 fajas de 45m (0,45 ha), plantadas con lapacho (Handroanthus heptaphyllus), orientación E-O.

Figura 2. Croquis de las parcelas de enriquecimiento.

Sketch of the plots enrichment. 
- Sobrevivencia: planta viva o muerta, luego del sexto año de plantación.

- Diámetro normal: diámetro medido a 1,3 metros de altura, con cinta diamétrica, medición realizada luego del sexto año de la plantación.

- Altura total (H): medida con una vara con marcas cada $25 \mathrm{~cm}$, medición realizada luego del sexto año de la plantación.

- Situación de competencia. Ubicado el observador junto a la planta evaluada, se observó hacia arriba y dividiendo en cuatro cuadrantes, se clasificó en cuatro categorías subjetivas (figura 3):

- Situación 1, sin presencia de cobertura.

- Situación 2, con presencia de alguna rama del estrato superior, cubriendo no más de un cuadrante.

- Situación 3, más de un cuadrante ocupado y menos de cobertura completa.

- Situación 4, cobertura completa.

Esta escala cualitativa se contrastó con los valores de la relación altura total/dap, tomando a la misma como una cuantificación de la situación individual de competencia donde se desarrolla cada individuo. Esta relación adopta valores mayores cuando es mayor la competencia.

- Daños: los daños se clasificaron en:

- Daños producido por cérvidos nativos llamados “guazuncho” (Mazama gouazoubira Fischer) al raspar sus cuernos en el tallo de las plantas jóvenes, clasificados según su intensidad en leves (lesiones que afectan una sola cara del fuste) moderadas (lesiones que afectan más de una cara del fuste) y severas (lesiones que provocan el anillado del fuste).

- Daños producidos por hormigas (Acromyrmex $s p)$, se estimó un porcentaje de defoliación de la copa.

- Tallos quebrados a distintas alturas, sin poder atribuir a una causa en particular (posiblemente corte involuntario con machete durante el desmalezado de limpieza de la faja, especialmente en plantas pequeñas).

Influencia de la orientación y de la competencia en el crecimiento. Para la especie Peltophorum dubium, se analizó estadísticamente el efecto de la orientación de las fajas y la influencia de la situación de competencia en el crecimiento en diámetro y altura. Se utilizó un diseño de muestreo jerárquico o anidado, en el que las fajas constituyen submuestras de cada orientación (NS y EO). Estas submuestras son tres fajas con cinco parcelas de tres individuos cada una.

Análisis estadístico. Se efectuó el análisis de la varianza para las variables diámetro normal y altura total de Peltophorum dubium, conforme el siguiente modelo matemático:

$$
Y_{i j}=u+S c_{i}+f(O)_{j(i)}+E_{i j}
$$

Donde:

$\mathrm{Y}_{\mathrm{ij}}$ : es la variable registrada (diámetro normal y altura total). u: es la media de la población.

$\mathrm{Sc}_{\mathrm{i}}$ : es el efecto de la situación de competencia

$\mathrm{f}(\mathrm{O})_{\mathrm{j}(\mathrm{i})}$ : es el efecto de la orientación dentro de la situación de competencia.

$E_{i j}$ : es el error.

Posteriormente se utilizó la situación de competencia como covariable y se probaron diferencias de medias entre las diferentes situaciones de competencia.

Supuestos paramétricos. Los resultados de la distribución de los residuos y la homogeneidad de varianzas, no evidenciaron discrepancias con los supuestos paramétricos. Se verificaron la normalidad de los residuos y ligera heterogeneidad de varianzas tanto en la variable diámetro normal como en altura total.

\section{RESULTADOS}

Sobrevivencia. Luego del quinto año de plantación, el promedio general de sobrevivencia fue de 51 \% (cuadro 1 ). La especie Grevillea robusta tuvo la menor sobrevivencia, mientras que Peltophorum dubium fue la que presentó mayor sobrevivencia.

El número de plantas que superó $1,3 \mathrm{~m}$ de altura fue prácticamente igual a la cantidad de plantas que sobrevivieron al quinto año en el caso de Peltophorum dubium (141 plantas). Las otras especies presentaron mayor diferencia entre la cantidad de plantas que superaron el 1,3 m de altura (cuadro 1) y el número de plantas vivas al quinto año de plantación (Grevillea robusta con 71 plantas vivas y Handroanthus heptaphyllus con 85 plantas vivas).

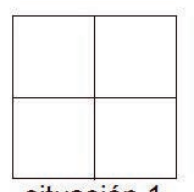

situación 1

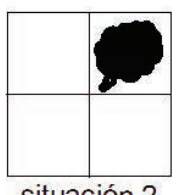

situación 2

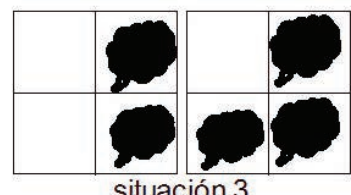

situación 3

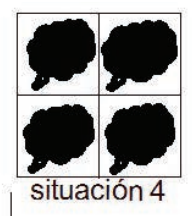

Figura 3. Representación de las distintas situaciones de competencia.

Representation of the different competition situations. 
Los ejemplares de Peltophorum dubium fueron los que tuvieron mayor desarrollo tanto en altura como en diámetro. El menor crecimiento se observó en Handroanthus heptaphyllus, lo cual era esperable por ser una especie de crecimiento lento (cuadro 1).

Los incrementos medios anuales de Grevillea robusta de $0,56 \mathrm{~cm}$ en dap y $0,77 \mathrm{~m}$ en altura, así como los de Peltophorum dubium, 0,78 cm en dap y 0,97 $\mathrm{m}$ en altura, fueron relativamente bajos.

Daños de animales. En las plantas de Peltophorum dubium, el principal daño observado fue el producido por cérvidos, el $40 \%$ de los individuos presentó lesiones menores; el 10,5 \% daños severos (anillado). Estos daños no se observaron en las otras especies.

Grevillea robusta presentó ataques de hormigas (Acromyrmex sp.), con el $40 \%$ de las plantas defoliadas al segundo y al quinto año; en Peltophorum dubium y Handroanthus heptaphyllus no se encontraron daños que se puedan asociar a hormigas.

Se encontraron en forma recurrente plantas de Handroanthus heptaphyllus con los tallos quebrados a distintas alturas, sin poder atribuirlo a una causa en particular. Esta situación no fue observada en individuos de las otras especies estudiadas.

Nivel de competencia. La estimación de la situación de competencia expresada mediante la relación altura total/ dap, como se indica en el cuadro 2, refleja la condición de crecimiento individual de las plantas jóvenes de Peltophorum dubium. En relación a las plantas de Grevillea robusta esta condición está perjudicada por la alta tasa de mortalidad registrada (64 \%).
Influencia de la orientación de las fajas y de la situación de competencia en el diámetro de Peltophorum dubium. La variable orientación no tuvo efectos significativos sobre el diámetro de las plantas de $P$. dubium, en las orientaciones analizadas $(P>0,05)$.

Para la misma variable, utilizando la situación de competencia como covariable, el modelo no identificó a la orientación como fuente de variación significativa $(P>0,05)$, mientras que sí detectó efectos altamente significativos de las situaciones de competencia $(P<0,05)$. La prueba de diferencias de medias mostró que el diámetro medio en la situación de competencia 1 fue significativamente mayor al diámetro medio en las demás situaciones de competencia (cuadro 3).

Influencia de la orientación y de la situación de competencia en la altura de Peltophorum dubium. Tampoco se detectó que la orientación de la faja de enriquecimiento fuese

Cuadro 3. Medias del dap para las diferentes situaciones de competencia (Duncan, alfa $=0,05$ ).

Means of dbh for various competition situations (Duncan, alfa $=0.05)$.

\begin{tabular}{ccc}
\hline Situación de competencia & dap $(\mathrm{cm})$ & N \\
\hline 4 & $3,23^{\mathrm{a}}$ & 26 \\
3 & $3,46^{\mathrm{a}}$ & 13 \\
2 & $3,54^{\mathrm{a}}$ & 13 \\
1 & $5,04^{\mathrm{b}}$ & 20 \\
\hline
\end{tabular}

Letras distintas indican diferencias significativas $(P \leq 0,05)$.

Cuadro 1. Sobrevivencia y desarrollo de las plantas de enriquecimiento al quinto año de plantación.

Survival and development of enrichment plants five years after planting.

\begin{tabular}{lcccc}
\hline \multicolumn{1}{c}{ Especie } & $\mathrm{N}^{*}$ & Sobrevivencia (\%) & dap $\pm \mathrm{S}(\mathrm{cm})$ & Altura total $\pm \mathrm{S}(\mathrm{m})$ \\
\hline Peltophorum dubium & 140 & 71 & $3,72 \pm 1,83$ & $5,17 \pm 1,73$ \\
Grevilllea robusta & 60 & 36 & $1,98 \pm 1,16$ & $3,60 \pm 1,39$ \\
Handroanthus heptaphyllus & 40 & 43 & $0,63 \pm 0,28$ & $0,80 \pm 0,56$ \\
\hline
\end{tabular}

*Valores de $\mathrm{N}$ = corresponden a las plantas que superaron la altura de 1,3 m y por lo tanto contaban con valor de dap.

Cuadro 2. Relación altura total/diámetro (H/dap) por especie y situación de competencia.

Height/diameter relation (H/dap) by species and competition situation.

\begin{tabular}{lcccc}
\hline \multirow{2}{*}{ Especie } & \multicolumn{4}{c}{ Situación de competencia (media \pm S) } \\
\cline { 2 - 4 } & 1 & 2 & 3 & 4 \\
\hline Peltophorum dubium & $130 \pm 0,26$ & $148 \pm 0,21$ & $160 \pm 0,23$ & $175 \pm 0,35$ \\
Grevillea robusta & $185 \pm 0,44$ & $173 \pm 0,34$ & $263 \pm 0,38$ & $229 \pm 0,50$ \\
\hline
\end{tabular}


una fuente de variación significativa en el crecimiento en altura total de los individuos $(P>0,05)$.

$\mathrm{Al}$ analizar el efecto de las orientaciones sobre la altura para cada situación de competencia, se encontró que solo para la situación sin competencia el efecto de las orientaciones fue significativo $(P>0,05)$, siendo mayor la media correspondiente a la orientación Este-Oeste (6,6 m) que la orientación Norte-Sur (5,8 m).

\section{DISCUSIÓN}

El promedio general de sobrevivencia logrado en el ensayo de enriquecimiento es un valor bajo aunque semejante a lo observado por Eibl et al. (1993) para Peltophorum dubium, $54 \%$ al cuarto año de plantación. La escasa sobrevivencia observada tanto para Grevillea robusta, como para Handroanthus heptaphyllus pudieron estar relacionados con daños producidos por hormigas en el primer caso y daños mecánicos en el segundo caso. Sobre la sobrevivencia de $P$. dubium influyeron los daños provocados por guazunchos, sin embargo, fueron superiores a los obtenidos por Eibl et al. (1998) de $30 \%$ al séptimo año y los informados por Vera y Gauto (2002), quienes obtuvieron un $65 \%$ de sobrevivencia al año de plantación. Senilliani et al. (2006) registraron al sexto año de plantación, valores similares de sobrevivencia (70 \%) a los encontrados en este trabajo, para un enriquecimiento en bosquetes de $P$. dubium en el mismo tipo de bosque.

Con relación a los daños observados en las plantas, la mayoría son factibles de controlar mediante protección individual y control de insectos. Esto sin dudas incrementaría los costos de mantenimiento, que para diferentes autores constituye uno de los principales inconvenientes de este método de enriquecimiento (Lamprecht 1990, Montagnini et al. 1997, Grulke et al. 2007, Brassiolo et al. 2013).

El crecimiento en diámetro y en altura coincide con lo observado a partir de la sobrevivencia, ya que $P$. dubium fue la especie que tuvo mejor desarrollo, mientras que posiblemente los daños que tuvieron las otras especies influyeron sobre su crecimiento.

Los valores registrados para $P$. dubium fueron semejantes a los observados por Montagnini et al. (1997) en un bosque degradado de la selva misionera (incremento medio anual de $0,74 \mathrm{~cm}$ en dap y de $0,77 \mathrm{~m}$ en altura total) pero inferiores a los observados por Pérez (2000) quien obtuvo un incremento de $1,18 \mathrm{~cm} /$ año en reforestaciones en macizo en Formosa y a los obtenidos en un enriquecimiento en bosquetes (incremento medio de dap 1,27 cm por año), en un bosque alto degradado del chaco húmedo, en rodales contiguos al sitio donde se emplaza el ensayo del presente estudio y donde se realizaron raleos para liberar de competencia a las plantas de enriquecimiento (Senilliani et al. 2006).

La respuesta en el crecimiento de las plantas de $P$. $d u$ bium está influenciada por el efecto de la situación de competencia que mostró valores altamente significativos. Esto coincide con lo observado por Montagnini et al. (1997) quienes atribuyen el pobre desarrollo de los ejemplares de esta especie a la competencia.

La orientación de la faja, que según Lamprecht (1990) y Wadsworth (2000) es uno de los factores que mejora las condiciones de luminosidad de las plantas de enriquecimiento, solo tuvo influencia sobre el crecimiento en diámetro y altura de $P$. dubium cuando las plantas se desarrollaron con bajo nivel de competencia (situación de competencia 1). Esto coincide con lo encontrado en Paraguay por Quinteros y Ortíz (1999) durante los primeros años del enriquecimiento.

La importancia de las situaciones de competencia (diferencia altamente significativa) muestra que en el caso de $P$. dubium se podría lograr mejor crecimiento de los individuos plantados con prácticas que permitan reducir la competencia, tales como mayor ancho de fajas coincidiendo con lo planteado por Montagnini et al. (1997) o eliminando la cobertura sobre las plantas de enriquecimiento.

\section{CONCLUSIONES}

De las especie analizadas, $P$. dubium presenta el mejor desempeño, aunque con problemas de sobrevivencia. Pese a que $G$. robusta y $H$. heptaphyllus muestra baja sobrevivencia y elevados niveles de daño, considerando la alta calidad de sus maderas deben ser incluidos en futuros trabajos de enriquecimiento.

A pesar de que $P$. dubium presenta los mejores valores de crecimiento tanto en diámetro como altura, los mismos son inferiores a los obtenidos con mejores condiciones de luminosidad. Los resultados del ensayo sugieren que en el caso de $P$. dubium la orientación de la faja no es suficiente para mejorar las condiciones de luminosidad de las plantas de enriquecimiento, por lo que se recomienda utilizar fajas más anchas, o introducir raleos destinados a disminuir la competencia sobre las plantas de enriquecimiento.

\section{AGRADECIMIENTOS}

Agradecemos a la Secretaría de Ciencia y Técnica de la Universidad Nacional de Santiago del Estero por el apoyo financiero para la realización del trabajo. Además estamos especialmente agradecidos con Carlos López por su ayuda en el análisis estadístico de los datos y con Analía Guzmán por su constante apoyo. Dos revisores anónimos mejoraron sustancialmente la versión original del presente trabajo.

\section{REFERENCIAS}

Brassiolo M, M Abt, M Grulke. 2013. Prácticas forestales en los bosques nativos de la República Argentina, Región del Parque Chaqueño. Consultado 15 feb. 2014. disponible en http://www.ambiente.gob.ar/?idarticulo=10930

Cabrera AL. 1976. Regiones fitogeográficas de la República Argentina. Fasc. 1. Enciclopedia Argentina de la Agricultura y 
la Jardinería. 2a ed. Buenos Aires, Argentina. ACME. 85 p.

Eibl B, F Montagnini, L Grance, D Maiocco, D Nozzi. 1998. Técnicas de enriquecimiento de bosques degradados en la selva subtropical paranaense de Misiones Argentina. In Actas VI Jornadas Técnicas Forestales Ecología de especies nativas de la selva subtropical Misionera. Misiones, Argentina. FCF - UNM. p. 36-44.

Eibl B, F Morandi, D Muñoz, L Martínez. 1993. Enriquecimiento en fajas con especies forestales nativas en San Pedro. Misiones. In Actas VII Jornadas Técnicas Forestales; “Ecosistemas forestales nativos, uso, manejo y conservación”. Misiones, Argentina. FCF - UNM. p. 268-277.

FAO (Food and Agriculture Organization of the United Nations, IT). 2010. Evaluación de los recursos forestales mundiales 2010. 247 p. (Estudio FAO Montes 163).

Gobierno de la provincia de Chaco. 2006. Inventario Forestal 2005: provincia de Chaco. Resistencia, Argentina, Ministerio de la Producción, Librería de la Paz. 148 p.

Gómez C. 2010. Influencia de diferentes métodos de raleo sobre la regeneración natural en el bosque alto del Chaco húmedo Tesis de Maestría en Cs. Forestales. Misiones, Argentina. Facultad de Ciencias Forestales, Universidad Nacional de Misiones. 62 p.

Grulke M, M Brassiolo, G Soto, F Lannes, K Obst, J Michela. 2007. Manual para el manejo forestal sustentable de los bosques nativos de la provincia del Chaco. Resistencia, Argentina. Ministerio de Producción, provincia del Chaco. 216 p.

INTA (Instituto Nacional de Tecnología Agropecuaria, AR) GPC (Gobierno de la Provincia de Chaco, AR). 1997. Carta de suelos de la República Argentina, No 15. Pcia. de Chaco. Los suelos del departamento Presidencia de La Plaza. 326 p.

Lamprecht HL. 1990. Silvicultura en los trópicos. Eschborn, Alemania. GTZ. 335 p.

Montagnini F, B Eibl, L Grance, D Maiocco, D Nozzi 1997. Enrichment planting in overexploited subtropical forests of the Paranaense region of Misiones, Argentina. Forest Ecology and Management 99. 237-246.

Montagnini F, B Eibl, R Fernández, M Brewer. 2006. Estrategias para la restauración de paisajes forestales, experiencias en Misiones, Argentina. In Actas II Congreso Forestal Latinoamericano IUFRO. Talca, Chile.

Morello J, J Adámoli. 1974. Las grandes unidades de vegetación y ambiente del Chaco Argentino. Segunda parte: Vegetación y ambiente de la provincia del Chaco. Buenos Aires, Argentina. INTA. 130 p. (Serie Fitogeográfica $N^{\circ} 13$ ).

Olivares R, LA Valdés, JE Menendi. 1997. Recopilación y tratamiento de datos pluviométricos mensuales y anuales, período 1956-1996. Resistencia, Argentina. Ministerio de Agricultura Ganadería y Recursos Naturales, provincia de Chaco. 215 p.

Pérez V, 2000. Perspectivas de la forestación en la provincia de Formosa. In Resúmenes III Jornadas de Ciencia y Tecnología de la Universidad Nacional de Formosa. p. 52.

Quinteros M, M Vera de Ortiz. 1999. Enriquecimiento de bosque nativo degradado con cinco especies nativas y una especie introducida. Choré, departamento San Pedro, Paraguay. Informe Final Dirección de Investigación. Asunción, Paraguay. Universidad Nacional de Asunción. 23 p.

Sánchez J, I Götz, W Segovia. 1993. Enriquecimiento de bosques nativos. Implantación bajo cubierta. In Actas VII Jornadas Técnicas Forestales Ecosistemas forestales nativos. Uso, manejo y conservación. Misiones, Argentina. ISIF-FCFUNM. p. 300-308.

Senilliani MG, C Gómez, M Brassiolo, M Galindez. 2006. Enriquecimiento con Ibirá Puitá Guazú (Peltophorum dubium Spreng) en la Región del Chaco Sub Húmedo. In Actas $1^{\circ}$ Jornada taller nacional de protección y manejo sustentable del bosque nativo. Entre Ríos, Argentina. p. 125-132.

Vera N, O Gauto. 2002. Conducción de la regeneración natural y enriquecimiento en bosques degradados. In Actas IX Jornadas Técnicas Forestales. Misiones, Argentina. INTA-FCFMEYRNRYT. Artículo $\mathrm{N}^{\circ} 48$. 
